\title{
Enantio-divergent epoxidation of alkenes with a photo-switchable phosphate manganese-salen complex
}

Roeland Nolte ( $\sim$ R.Nolte@science.ru.nl )

Radboud University, Institute for Molecules and Materials, Nijmegen, The Netherlands

Xiaofei chen

Pieter Gilissen

Radboud University, Institute for Molecules and Materials, Nijmegen, The Netherlands https://orcid.org/0000-0003-2111-9564

Paul Tinnemans

Radboud University, Institute for Molecules and Materials, Nijmegen, The Netherlands

Nicolas Vanthuyne

Aix Marseille Univ

Floris Rutjes

Radboud University

Ben Feringa

University of Groningen

Johannes Elemans

Radboud University Nijmegen, Institute for Molecules and Materials

\section{Article}

Keywords:

Posted Date: February 1st, 2022

DOI: https://doi.org/10.21203/rs.3.rs-1253856/v1

License: (c) (i) This work is licensed under a Creative Commons Attribution 4.0 International License.

Read Full License

Version of Record: A version of this preprint was published at Nature Synthesis on September 22nd, 2022. See the published version at https://doi.org/10.1038/s44160-022-00157-7. 


\section{Abstract}

The development of enantio-divergent catalysts capable of producing in a controlled fashion each of the two enantiomeric products from one substrate, is highly challenging. It requires the implementation of a switching function into the catalyst, which can reversibly change the chiral reaction environment during catalysis. Here we report a photo-switchable phosphate ligand derived from 2,2'-biphenol, which axially coordinates as the counter ion to an achiral manganese(III) salen catalyst, providing the latter with the ability to dynamically switch stereoselectivity in the epoxidation of alkenes. Both enantiomers of the chiral ligand exist as a pair of pseudo-enantiomers, which can be efficiently interconverted by irradiation with light of different wavelengths. The opposite axial chiralities of these pseudo-enantiomers are efficiently transferred to the manganese(III) salen catalyst. With this switchable ion-paired catalyst, the enantioselectivity of the epoxidation of a variety of alkenes can be controlled, resulting in opposite enantiomeric excesses of the epoxide products. This dynamic transfer of chirality from a photoswitchable counter ion to a metal complex will greatly broaden the scope of supramolecular catalysts. In future studies this photo-switchable catalyst will be used to write digital information in the form of chiral epoxides on single polymer chains.

\section{Introduction}

Chirality is a hallmark of numerous molecules in nature, such as amino acids, sugars, proteins, and DNA. ${ }^{1}$ In cells, enzymes precisely control life processes by carrying out strictly enantioselective catalytic transformations on bound substrates. ${ }^{2}$ Taking the stereochemical fidelity of such processes as a blueprint, great advances have been made in stereoselective syntheses in academic laboratories and pharmaceutical industries. Although many chiral catalysts capable of producing close to enantiomerically pure products have been developed, the design of enantio-divergent catalysts, which are capable of producing both enantiomeric products of a reaction via a switching function, is still an intriguing challenge. ${ }^{3-6}$ To achieve controllable switching, a specific design of molecules that can exist in various distinct conformations is required. In 1999, the first example of a molecular motor displaying light- and heat-controlled unidirectional rotating behaviour, was reported. ${ }^{7}$ It relies on the cis-trans isomerization of an overcrowded alkene located between an aromatic stator and rotor fragment, which is accompanied by a change in the relative helicity of the fragments, adopting either an $M$ or a $P$ configuration. The use of light as a non-invasive external stimulus for the isomerization reaction excludes the undesired generation of chemical waste or the occurrence of side reactions. ${ }^{8}$ The first-generation molecular motors ${ }^{7}$ required both photochemical and thermal isomerization steps to interconvert the pseudo-enantiomers. In contrast, the later developed second-generation molecular motors are equipped with a symmetric fluorenyl stator and can be interconverted by solely utilizing light (Fig. 1a). ${ }^{9,10}$ Recent advances in constructing asymmetric organocatalysts with an implemented "on-off" function have drawn attention because they are capable of performing complicated tasks when they are triggered by external stimuli, such as light, changes in solvent or $\mathrm{pH}$, or the binding of metals. ${ }^{3,11-13}$ However, the development of enantio-divergent catalysts is still highly challenging, and only few examples of such systems have 
been reported. A first-generation molecular motor equipped with organocatalytic functions has been applied as a catalyst in an asymmetric Michael addition, producing a mixture of adducts with $50 \%$ e.e., while after a thermally activated reversal in helicity of the motor the adduct with an opposite enantioselectivity of $54 \%$ e.e. was preferentially obtained $(\Delta$ e.e. $=104 \%) .{ }^{14}$ First-generation molecular motor have also been used as a stereodivergent catalyst in Henry reactions ( $\Delta$ e.e. $=$ up to $116 \%),{ }^{15}$ as a ligand in a palladium-catalyzed desymmetrization reaction $(\Delta$ e.e. $=174 \%),{ }^{16}$ and as anion binding catalysts $(\Delta$ e.e. $=$ up to $142 \%) .{ }^{17} \mathrm{~A}$ second-generation molecular motor has been used as photoswitchable catalyst for the enantio-divergent addition of diethylzinc to aldehydes $(\Delta$ e.e. $=$ up to $113 \%) .{ }^{10}$ Recently, Leigh et al. reported a switchable molecular shuttle, which could stereoselectively catalyze the conjugate addition of aldehydes to vinyl sulfones, yielding products with opposite enantiomeric configurations $(\Delta$ e.e. $=$ up to $60 \%) .{ }^{18}$

Chiral phosphates as ligands or counter ions in ion-paired catalysts ${ }^{19}$ can be of importance in enantioselective catalysis, as they can strongly render and amplify chirality during the catalytic reaction at the metal center. ${ }^{20}$ In 2002, Lacour et al. reported pioneering work in which chiral hexacoordinated phosphate anions improved the enantioselectivity of an oxone-mediated epoxidation. ${ }^{21}$ More recently, chiral phosphates were employed in asymmetric Brønsted acid-based catalysis, ${ }^{22}$ and gold, ${ }^{23}$ palladium ${ }^{24}$ and iridium-based ${ }^{25}$ transition metal catalysis. A typical example of such a chiral phosphate is the 3,3'-bisaryl-substituted BINOL-derivative shown in Fig. $1 \mathrm{~b}$, which can provide enantioselectivity in catalytic reactions. ${ }^{26}$ List et al. reported a series of ion-paired catalysts in which chiral BINOL-based phosphate counter ions coordinated to a manganese(III) salen complex displayed excellent enantioselectivities (up to $96 \%$ e.e.) in the epoxidation of alkenes. ${ }^{27,28}$

Current work in our group aims at encoding binary information into polymer chains, by threading them through a cavity-containing catalyst that can write chiral epoxides, i.e. $(R, R)$-epoxide $=$ digit $1,(S, S)$ epoxide = digit $0 .{ }^{29}$ In order to make this writing stereo controllable, we set out to investigate enantiodivergent epoxidation catalysts that can be switched non-invasively, i.e. with light. Herein, we report ionpaired complex Mn2 (Fig. 1e), which consists of two components: an achiral manganese(III) salen complex (Fig. 1C), which is capable of catalytically transferring an oxygen atom from an oxidant to an olefin substrate to generate an epoxide, and a photoresponsive 2,2'-biphenol-derived phosphate ligand 1 (Fig. 1d), which coordinates to the manganese(III) center and induces chirality into the salen complex. Upon irradiation with light, the overcrowded alkene moiety of this counter ion switches helicity $(M$ or $P)$, thereby switching the axial chirality of the 2,2'-biphenol moiety $\left(R_{\mathrm{a}}\right.$ or $\left.S_{\mathrm{a}}\right)$, which in turn switches the chiral environment of the manganese(III) salen (Fig. 1e). As a result, the catalyst becomes capable of carrying out controlled enantio-divergent epoxidation reactions.

\section{Results And Discussion}

Design. The design of the photo-switchable molecular catalyst is inspired by the previously reported lightresponsive motors derived from 2,2'-biphenol (Fig. 1a). ${ }^{10,30}$ The aromatic fragments connected to the 
overcrowded alkene are synthesized separately, which allows for their separate modification. The biphenol-containing moiety contains a stereogenic center with a methyl substituent, which controls the unidirectionality of the rotation of the motor. ${ }^{31}$ Second-generation molecular motors composed of a symmetric fluorenyl stator and a six-membered ring rotor have been reported as excellent chiroptical switches, because of their high photostationary state (PSS) ratios, as well as for the thermal stability of their metastable iosmer $\left(\mathrm{t}_{1 / 2} \sim\right.$ years) ${ }^{32}$ In the case that the initial stereoisomer and the stereoisomer obtained by photochemical isomerization are both relatively stable, it becomes possible to use the motor as a two-state molecular switch, and by irradiation with light of two different wavelengths, the $M$ and $P$ helical configurations of the motor can be reversibly switched. These stable helicities may continuously transfer and amplify the chirality of a metal complex during catalytic reactions. ${ }^{10,30}$

The use of chiral manganese(III) salen derivatives as catalysts for the asymmetric epoxidation of alkenes has been extensively described by the groups of Jacobsen ${ }^{33}$ and Katsuki. ${ }^{34}$ Although a manganese(III) salen containing an ethylene-bridge is symmetric (Fig. 1c), and cannot be used as an asymmetric catalyst itself, the axial coordination of a chiral counter ion to the metal center can induce a chiral configuration, leading to high enantioselectivities in oxidation reactions. ${ }^{27,28,35}$ Simultaneously, such a coordination can lead to an increase in reactivity of the catalyst. ${ }^{36}$ We envisaged that, after deprotonation, the phosphoric acid-functionalized molecular switch 1 can act as an anionic chiral inducer ligand for a manganese(III) salen epoxidation catalyst, providing the resulting ion-paired complex Mn2 with inherent switchable chirality for the enantio-divergent epoxidation of alkenes.

Specifically, Mn2 includes five chiral elements (Fig. 1e): (1) the point chirality at the stereogenic center with the methyl substituent attached (magenta); (2) the helicity of the aromatic fragments connected to the overcrowded alkene (blue); (3) the bis-aryl axial chirality $\left(S_{\mathrm{a}}\right.$ or $\left.R_{\mathrm{a}}\right)$; (4) the dynamic helical geometry between the aromatic rings of the salen and switch ( $P$ or $M)$ (black); $(5)$ the chirality of the phosphorus atom after coordination of the phosphate group to the manganese(III) salen.

We reasoned that, upon its coordination to the manganese(III) center, a pure enantiomer of (deprotonated) 1 can transfer its helicity to the salen backbone and induce a specifically twisted conformation of the ethylene linker, thereby enabling $\mathrm{Mn} 2$ to enantioselectively epoxidize an alkene. Upon irradiation of the catalyst with light with a wavelength at which the overcrowded alkene absorbs (the $\pi \rightarrow \pi^{*}$ transition), the phosphate ligand is expected to be converted to its pseudo-enantiomer, thereby transferring its opposite helicity to the salen and providing the ethylene-bridge with an opposite twist. The catalyst is then expected to preferably catalyze the formation of the opposite enantiomer of the epoxide. In other words, the helicity of the chiral phosphate counter ion can be modulated with light, which, as a consequence, is directing the enantioselectivity of the Mn-salen catalyst towards either enantiomer of the chiral epoxide product externally and on demand.

Synthesis. The synthesis and resolution of ion-paired catalyst Mn2 is depicted in Fig. 2. The synthesis of the motor-containing phosphoric acid 1 started from commercially available 4-arylbutanoic acid $\mathbf{3}$, which was dibrominated with bromine at the ortho-positions with respect to the methoxy-substituent. An 
intramolecular Friedel-Crafts acylation of the crude dibromocarboxylic acid was accomplished with Eaton's reagent at mildly elevated temperature. The key synthetic step was the regioselective introduction of the tert-butylphenyl substituent onto aryl dibromide 4. The combination of $\mathrm{Pd}_{2} \mathrm{dba}_{3}$ as palladium source combined with the bulky bidentate phosphine ligand bis[(2-diphenylphosphino)phenyl] ether (DPEPhos) allowed us to obtain cross-coupled product 5 in good yield. 2-Bromoanisole 6 was crosscoupled under standard Suzuki-Miyaura conditions to afford the biphenyl derivative 7. ortho-Lithiation of compound 7 followed by a reaction of the aryl lithium species with trimethylborate and aqueous workup yielded boronic acid $\mathbf{8}$. A subsequent Suzuki-Miyaura reaction between bromide $\mathbf{5}$ and boronic acid $\mathbf{8}$ afforded cross-coupled compound 9 in very good yield. The stereogenic center was introduced by amethylation, furnishing ketone 10 in excellent yield. The reaction of ketone 10 with hydrazine monohydrate in $n$-butanol using scandium(III) triflate as the catalyst afforded hydrazone 11 . The latter compound was oxidized in situ using [bis(trifluoroacetoxy)iodo]benzene (PIFA), and the resulting diazo intermediate was reacted with freshly prepared thioketone $12 .{ }^{37}$ The Barton-Kellogg olefination initially afforded an episulfide intermediate, which was desulfurized by tris(dimethylamino)phosphine (HMPT), providing overcrowded alkene 13 in reasonable yield after this three-step sequence. Next, the methyl protecting groups were removed with methylmagnesium iodide, ${ }^{10}$ affording diol 14 in nearly quantitative yield. Racemic compounds 10-14 were all obtained as mixtures of two conformers due to the non-fixed axial chirality. The biaryl axis was fixed in a specific axial chirality once diol 14 was reacted with phosphoryl chloride, providing phosphoric acid $\mathbf{1}$ as a single conformer. Previous investigations on aryl substituted molecular motors and switches have shown that the aryl substituent in the rotor part is always parallel to the fluorenyl lower half. ${ }^{10,30,38}$ The enantiomers of 1 were resolved by preparative chiral HPLC (Supplementary Fig. 1-4). The structure of phosphoric acid 1 was unequivocally proven by NMR spectroscopy and mass spectrometry. Finally, the reaction of enantiopure or racemic phosphoric acid 1 with manganese(III) salen derivative $\mathrm{Mn}^{159}$ afforded the enantiopure or the racemic ion pairs Mn2, respectively.

Photochemical isomerization. We first investigated the switching properties of the enantiopure phosphoric acid ligand isomer $\left(S, M, R_{\mathrm{a}}\right)-1$ and the corresponding ion-paired complex $\left(S, M, R_{\mathrm{a}}\right)-\mathrm{Mn} 2$ (Fig. 3a,b). The $\pi \rightarrow \pi^{*}$ transition of $\left(S, M, R_{\mathrm{a}}\right)-1$ was determined to be at $\lambda_{\max }=356 \mathrm{~nm}$ (Fig. 3c). Hence, we could accomplish the photochemical isomerization of stable $\left(S, M, R_{\mathrm{a}}\right)-1$ to metastable $\left(S, P, S_{\mathrm{a}}\right)-1$ with a LED emitting UV-light at $\lambda_{\max }=365 \mathrm{~nm}$ (Fig. 3c). The isomerization was accompanied by a bathochromic shift $(\Delta \lambda=43 \mathrm{~nm})$ of the main absorption band at $356 \mathrm{~nm}$, which is indicative of an increase in strain in the original alkene double bond after irradiation. ${ }^{32}$ The presence of an isosbestic point at $\lambda=379 \mathrm{~nm}$ indicates a clean unimolecular process. The Cotton effect associated with the main absorption band (positive CD signal at $\lambda=356 \mathrm{~nm}$ ) was inverted (negative CD signal at $\lambda=399 \mathrm{~nm}$ ), indicating an inversion of helicity, and an inversion of the $C D$ sign at $\lambda=300 \mathrm{~nm}$ was observed. When the resulting PSS $_{365}$-mixture containing metastable $\left(S, P, S_{\mathrm{a}}\right)-\mathbf{1}$ was irradiated with visible light at $\lambda=470 \mathrm{~nm}$, the opposite spectral changes were observed while the isosbestic point at $\lambda=379 \mathrm{~nm}$ was retained (Fig. $3 \mathrm{~d}$ ). To investigate the reversibility and stability of the switch, we subjected the solution of $\left(S, M, R_{\mathrm{a}}\right)-1$ to a 
series of isomerization cycles (Fig. 3e), and monitored the absorption maxima of the stable isomer $\left(S, M, R_{\mathrm{a}}\right)-1(\lambda=356 \mathrm{~nm})$, the metastable isomer $\left(S, P, S_{\mathrm{a}}\right)-\mathbf{1}(\lambda=399 \mathrm{~nm})$, and the $C D$ signal at $\lambda=300 \mathrm{~nm}$. All these maxima showed no signs of switching fatigue after five cycles.

The switching processes of 1 were investigated in more detail with the help of NMR spectroscopy (Fig. 4). To this end, we irradiated a solution of stable rac-1 (Fig. 4b) in situ with UV-light of $\lambda=365 \mathrm{~nm}$ for $45 \mathrm{~min}$ at $20^{\circ} \mathrm{C}$. A new set of signals appeared in the NMR spectrum, corresponding to the metastable isomer of rac-1 (Fig. 4c). Comparison of the integrals of the ${ }^{1} \mathrm{H}$ NMR resonances of the stable and metastable isomers allowed the determination of the photostationary state: $\mathrm{PSS}_{365}$ metastable:stable $\approx 95: 5$. This PSS-ratio is higher than that of similar second-generation molecular motors. ${ }^{10,30}$ Noticeably, the NMR spectrum of metastable rac-1 recorded after the solution had been stored under ambient conditions for two weeks was nearly identical to the spectrum that was recorded directly after the irradiation process (Fig. 4d), illustrating that the metastable state possesses a high thermal stability as well as an insensitivity to ambient light. When the sample was subsequently irradiated with visible light $(\lambda=470 \mathrm{~nm})$ at $20^{\circ} \mathrm{C}$ for $18 \mathrm{~h}$, the stable state was regenerated nearly quantitatively: $\mathrm{PSS}_{470}$ metastable:stable $\approx 5: 95$ (Fig. 4e).

In a next series of experiments, we investigated the photoisomerization processes of ion-paired catalyst Mn2 with UV-vis and CD spectroscopy (Fig. 3b). The use of ${ }^{1} \mathrm{H}$ NMR spectroscopy was not possible due to the paramagnetic nature of the compound. The changes in the UV-vis spectra of Mn2 upon photochemical isomerization with UV-light at $\lambda=365 \mathrm{~nm}$ (Fig. 3f, top) were reminiscent of those observed for phosphoric acid 1, i.e. a bathochromic shift of the absorption maximum of the overcrowded alkene moiety, and a clear isosbestic point at $\lambda=381 \mathrm{~nm}$. The CD spectrum of $\left(S, M, R_{\mathrm{a}}\right)-\mathrm{Mn} 2$ revealed that the chiral information in the phosphate ligand is effectively transferred to the manganese salen moiety, which is evidenced by the CD absorption at $\lambda=430 \mathrm{~nm}$ (Fig. 3f, bottom). Upon photochemical isomerization, the CD sign of $\mathrm{Mn} 2$ at $\lambda=300 \mathrm{~nm}$ changed sign from positive to negative, akin to what was observed for phosphoric acid 1. More importantly, the inversion of the CD sign at $\lambda=430 \mathrm{~nm}$ indicated that the chirality of the manganese(III) salen moiety could be effectively tuned by the switchable phosphate ligand. The reverse photochemical isomerization of the metastable ion pair $\left(S, P, S_{\mathrm{a}}\right)-\mathrm{Mn2}\left(\mathrm{PSS}_{365}\right)$ to the stable ion pair $\left(S, M, R_{\mathrm{a}}\right)-\mathrm{Mn} 2$ was accomplished by irradiation with visible light at $\lambda=470 \mathrm{~nm}$ (Fig. $3 \mathrm{~g}$ ). The isomerization processes of $\mathrm{Mn} 2$ were reversible for at least five cycles (Fig. 3h), which agrees with the behavior that was observed for phosphoric acid 1. Although quantitative information about the PSS-ratios could not be obtained from the above spectroscopic investigations, we reason that the $\mathrm{PSS}_{365}$ and $\mathrm{PSS}_{470}$-ratios of 1 and $\mathrm{Mn} 2$ are very similar, since their differences in absorbance at $\lambda=356(\Delta \mathrm{Abs} \approx 0.2)$ and $399 \mathrm{~nm}(\Delta \mathrm{Abs} \approx 0.2)$ and $\mathrm{CD}$ intensity at $\lambda=300 \mathrm{~nm}(\Delta \mathrm{CD} \approx 35$ mdeg) between the stable and metastable states are nearly identical under the same conditions.

X-ray crystallography. Besides obtaining spectroscopic evidence for the photochemical isomerization processes of $\mathrm{Mn2}$, we also investigated these by determining the crystal structures of $\mathrm{Mn} 2$ in the stable and metastable states. A single crystal of rac-Mn2 could be obtained by the slow evaporation of a 
solution of the complex in toluene- $n$-heptane $(1: 8, \mathrm{v} / \mathrm{v})$. From this crystal the X-ray structure of the complex could be determined (Supplementary Fig. 5). The unit cell of the crystal contains both enantiomers of the complex, i.e. $(S, M, R a)-M n 2$ and $(R, P, S a)-M n 2$. They display opposite helicities and axial chiralities of the overcrowded alkene axis and the biaryl moiety, respectively. In addition, they possess opposite fixed point chirality at the stereogenic center to which the methyl substituent is attached. The distance between the manganese atom and the phosphate oxygen atom is $2.04 \AA$, which indicates that the phosphate acts as an anionic ligand, which is strongly attached to the manganese center. Consequently, it may be expected that the chirality of the switch connected to the phosphate will be transferred to the manganese(III) salen, providing it with the ability to carry out asymmetric catalysis. In Fig. 5 the X-ray crystal structure of $\left(S, M, R_{\mathrm{a}}\right)-\mathrm{Mn} 2$ (present in the crystal of the racemic compound, Fig. 5a) is compared with the X-ray structure of the compound that is formed from it after light irradiation, i.e. $\left(S, P, S_{\mathrm{a}}\right)$-Mn2 (Fig. 5b). The comparison reveals that after light irradiation not only the helicity of the overcrowded alkene (blue color) changes, but also the twist in the ethylene bridge (magenta color) of the salen ligand. These chirality changes are thought to lie at the origin of the observed photo-switchable enantioselective epoxidation catalysis (vide infra).

Switchable asymmetric catalysis. Having established the light-switching properties of Mn2 by spectroscopic and X-ray crystallographic techniques, we employed the catalyst in the switchable epoxidation of alkenes. Using the chromene derivative 17 as a model substrate, iodosyl benzene as the oxygen donor, and $\left(S, M, R_{\mathrm{a}}\right)-\mathrm{Mn} 2$ as the catalyst in dry benzene, the alkene was converted nearly quantitatively and the enantiomers of the epoxide product were isolated in a combined yield of $89 \%$. Analysis of the mixture by chiral HPLC revealed that the two epoxide enantiomers were formed in a ratio of 13:87 (e.e. $=+74$ ) (Table 1, entry 1 and Fig. 6 b). In a separate experiment, we first irradiated the stable catalyst $\left(S, M, R_{\mathrm{a}}\right)-\mathrm{Mn} 2$ with light $\left(\lambda=365 \mathrm{~nm}\right.$ for $30 \mathrm{~min}, \mathrm{c}=1 \mathrm{mg} / \mathrm{mL}$ in $\left.\mathrm{CH}_{2} \mathrm{Cl}_{2}\right)$ to generate metastable catalyst $\left(S, P, S_{\mathrm{a}}\right)-\mathrm{Mn} 2$ after removal of the solvent. Using this catalyst in the epoxidation of $\mathbf{1 7}$ gave a combined epoxide yield of $73 \%$, but now in an opposite enantiomeric ratio of 71:29 (e.e. $=-42$ ) as revealed by chiral HPLC (Table 1, entry 2 and Fig. 6d). These experiments confirm that the phosphate ligand is indeed capable of efficiently transferring its chirality to the manganese(III) salen, and that lightinduced switching of the helicity of the ligand leads to switching of the enantioselectivity of the catalyst.

We found that catalytic reactions in freshly dried and distilled benzene and the use of $2.5 \mathrm{~mol} \%$ of Mn2 catalyst with respect to the substrate gave the most optimal results (Supplementary Table 2). The presence of water in the benzene solution turned out to have a negative impact on catalyst performance, both in terms of yield and stereoselectivity (Supplementary Table 3). We attribute this decrease in performance to a weakening of the coordination bond between the manganese center and the phosphate ligand, resulting in a decrease in activation of the metal center as well as a decrease in chirality transfer from the ligand to the salen backbone. A further decrease in solvent polarity by the addition of up to $50 \%$ $(\mathrm{v} / \mathrm{v})$ of $n$-heptane to the benzene solution did not improve the conversion nor the extent of enantioselectivity (Supplementary Table 4), neither did variations in reaction temperature (Supplementary Table 5). 
We then examined the absolute configurations of catalysts Mn2 by using the chiral Jacobsen manganese(III) salen complexes $(M, R, R)-16$ and $(P, S, S)$-16 as reference catalysts for the epoxidation of alkene 17 (Fig. 6). ${ }^{40}$ When $(M, R, R)-16$ was used as the catalyst, the trend in enantioselectivity of the epoxidation was the same as that observed when $\left(S, M, R_{\mathrm{a}}\right)-\mathrm{Mn} 2$ was used (Figs. $6 \mathrm{a}$ and $6 \mathrm{~b}$ ). Catalysts $(P, S, S)-16$ and $\left(S, P, S_{\mathrm{a}}\right)-\mathrm{Mn} 2$ showed the reverse trend (Figs. 6c and $\left.6 \mathrm{~d}\right)$, as expected. The key origin for the formation of a preferred chiral configuration of the epoxide is the specific induced chirality present in the salen ligand. The fact that $(M, R, R)-16$ and $\left(S, M, R_{\mathrm{a}}\right)-\mathrm{Mn} 2$ display the same trend in enantioselectivity indicates that they share a similar $M$ twist in their ligand backbone, while $(P, S, S)-16$ and $\left(S, P, S_{\mathrm{a}}\right)-\mathrm{Mn} 2$ share a $P$ twist. Based on the crystal structure of rac-Mn2 (Fig. 3), we thus can assign the first eluted fraction from the chiral HPLC to $\left(S, M, R_{\mathrm{a}}\right)-1$ and the second to $\left(R, P, S_{\mathrm{a}}\right)-1$ (Fig. 2).

To investigate the scope of the reactions catalysed by Mn2, we investigated the enantio-divergent epoxidation with different types of alkenes (Table 1). Chromene derivatives with various substituents (e.g. $\mathrm{NO}_{2}, \mathrm{CN}, \mathrm{Ph}, \mathrm{OAc}$ ) were smoothly converted into their corresponding epoxides, and so were various internally conjugated and terminal olefins. In all cases, light-induced switching of the catalyst resulted in opposite enantioselectivities of the epoxidation. For substrate 17, we also investigated the catalytic performance of the other enantiomer, $\left(R, P, S_{\mathrm{a}}\right)$-Mn2. This catalyst (Table 1, entry 3$)$ and its irradiated metastable isomer $\left(R, M, R_{\mathrm{a}}\right)$-Mn2 (Table 1, entry 4 ) gave epoxide product 17a in opposite stereoselectivities as compared to $\left(S, M, R_{\mathrm{a}}\right)-\mathrm{Mn} 2$ and $\left(S, P, S_{\mathrm{a}}\right)-\mathrm{Mn} 2$, respectively.

In all cases, catalysts $\mathrm{Mn} 2$ in their stable states produced epoxides with higher enantiomeric excesses than their metastable isomers that were obtained after irradiation with light of $\lambda=365 \mathrm{~nm}$. We attribute this difference to two factors. Firstly, the photochemical isomerization is not complete, i.e. the $\mathrm{PSS}_{365^{-}}$ ratio metastable:stable isomer is not 100:0. Secondly, the phosphorus atom of the phosphate moiety becomes intrinsically chiral once one of its oxygen atoms coordinates to the manganese center. If the oxygen-manganese bond remains intact during the isomerization process, i.e. the phosphorus atom retains its chirality, the stable and metastable complexes no longer are pseudo-enantiomers, but rather behave as diastereomers and a difference in e.e. is expected. However, the X-ray structures shown in Fig. 5 and in the Supplementary Figs. 5,6 and Supplementary Table 6 indicate that the stable and the metastable complexes are pseudo-enantiomers, meaning that the manganese-oxygen bond becomes broken during the photo-isomerization process and that the phosphorus center inverts its absolute stereochemistry. It should be noted that this reasoning is based on the solid-state X-ray structures and that the described situation may be different for the structures in solution. Further research on the structural properties of the ion-paired catalysts and their impact on the mechanism and stereoselectivity of the catalytic reactions are currently ongoing.

\section{Conclusion}

An ion-paired catalyst consisting of a photo-responsive phosphate anion coordinated to a Mn-salen has been developed and shown to exhibit significant levels of enantioselectivity in the epoxidation of alkenes. 
Irradiation of the biphenol-derived molecular motor connected to the phosphate changes its helicity and transfers its specific chirality to the salen backbone, governing the stereoselectivity during catalysis. The epoxidation of a variety of alkenes revealed that internal alkenes are more prone to enantioselective epoxidation than terminal ones, with the highest $\Delta$ e.e. value found being $116 \%$. We envision that the use of switchable photo-responsive ligands as chirality inducers, as shown here, may be widely applicable in transition metal catalysis and may significantly broaden the chiral pool for the development of more complex and efficient catalytic systems.

The current catalytic system will be attached to a macrocyclic compound and used in the switchable enantio-divergent epoxidation of polymers containing alkene double bonds. In this way we hope to write digital information, i.e. $(R, R)$ - and $(S, S)$ - epoxides (digits 1 and 0$)$ into single polymer chains. ${ }^{29}$ In this context we will try to further improve the currently achieved $\Delta$ e.e. value of $116 \%$ (Table 1 ) by optimizing the and adapting the ligand design. Nevertheless, the enatioselectivity values are probably good enough for writing as many error correcting programs have already been developed and are being used in the storage of information on DNA chains. ${ }^{29}$

\section{Declarations}

\section{Data availability}

The authors declare that the data supporting the findings of this study are included in the paper and its Supplementary Information files. Any further relevant data are available from the corresponding authors on request.

Author contributions. R.J.M.N. conceived the project. P.J.G. and F.P.J.T.R. designed the synthesis of compound 1. P.J.G. carried out the synthesis of 1 and investigated the photochemical properties of compounds 1 and Mn2. X.C. performed the catalysis experiments and grew the crystals. P.T. determined and analysed the crystal structure. N.V. separated the enantiomers by chiral HPLC. B.L.F contributed with his knowledge on molecular photo-switches. R.J.M.N. and J.A.A.W.E. supervised the project. All authors discussed the results, and helped write and discuss the manuscript.

Acknowledgements. This work was financially supported by the European Research Council (ERC Advanced Grant No. 74092 to R. J. M. N. and ERC Advanced Grant No. 227897 to B.L.F.) and by the Dutch Ministry of Education, Culture, and Science (Gravitation program 024.001.035).

\section{References}

1. Bonner, W. A. Chirality and life. Origins Life Evol. Biosphere 25, 175-190 (1995).

2. Brady, D. \& Jordaan, J. Advances in enzyme immobilisation. Biotechnol. Lett. 31, 1639 (2009). 
3. Vlatković, M., Collins, B. S. \& Feringa, B. L. Dynamic responsive systems for catalytic function. Chem. Eur. J. 22, 17080-17111 (2016).

4. Dorel, R. \& Feringa, B. L. Photoswitchable catalysis based on the isomerisation of double bonds. Chem. Commun. 55, 6477-6486 (2019).

5. Göstl, R., Senf, A. \& Hecht, S. Remote-controlling chemical reactions by light: Towards chemistry with high spatio-temporal resolution. Chem. Soc. Rev. 43, 1982-1996 (2014).

6. Blanco, V., Leigh, D. A. \& Marcos, V. Artificial switchable catalysts. Chem. Soc. Rev. 44, 5341-5370 (2015).

7. Koumura, N., Zijlstra, R. W., Van Delden, R. A., Harada, N. \& Feringa, B. L. Light-driven monodirectional molecular rotor. Nature 401, 152-155 (1999).

8. García-López, V., Liu, D. \& Tour, J. M. Light-activated organic molecular motors and their applications. Chem. Rev. 120, 79-124 (2020).

9. Koumura, N., Geertsema, E. M., Van Gelder, M. B., Meetsma, A. \& Feringa, B. L. Second generation light-driven molecular motors. Unidirectional rotation controlled by a single stereogenic center with nearperfect photoequilibria and acceleration of the speed of rotation by structural modification. J. Am. Chem. Soc. 124, 5037-5051 (2002).

10. Pizzolato, S. F. et al. Central-to-helical-to-axial-to-central transfer of chirality with a photoresponsive catalyst. J. Am. Chem. Soc. 140, 17278-17289 (2018).

11. Van Dijk, L. et al. Molecular machines for catalysis. Nat. Rev. Chem. 2, 0117 (2018).

12. Ihrig, S. P., Eisenreich, F. \& Hecht, S. Photoswitchable polymerization catalysis: state of the art, challenges, and perspectives. Chem. Commun. 55, 4290-4298 (2019).

13. Romanazzi, G., Degennaro, L., Mastrorilli, P. \& Luisi, R. Chiral switchable catalysts for dynamic control of enantioselectivity. ACS Catal. 7, 4100-4114 (2017).

14. Wang, J. \& Feringa, B. L. Dynamic control of chiral space in a catalytic asymmetric reaction using a molecular motor. Science 331, 1429-1432 (2011).

15. Vlatković, M., Bernardi, L., Otten, E. \& Feringa, B. L. Dual stereocontrol over the Henry reaction using a light- and heat-triggered organocatalyst. Chem. Commun. 50, 7773-7775 (2014).

16. Zhao, D., Neubauer, T. M. \& Feringa, B. L. Dynamic control of chirality in phosphine ligands for enantioselective catalysis. Nat. Commun. 6, 6652 (2015).

17. Dorel, R. \& Feringa, B. L. Stereodivergent anion binding catalysis with molecular motors. Angew. Chem. Int. Ed. 59, 785-789 (2020). 
18. Dommaschk, M., Echavarren, J., Leigh, D. A., Marcos, V. \& Singleton, T. A. Dynamic control of chiral space through local symmetry breaking in a rotaxane organocatalyst. Angew. Chem. Int. Ed. 58, 1495514958 (2019).

19. Brak, K. \& Jacobsen, E. N. Asymmetric ion-pairing catalysis. Angew. Chem. Int. Ed. 52, 534-561 (2013).

20. Phipps, R. J., Hamilton, G. L. \& Toste, F. D. The progression of chiral anions from concepts to applications in asymmetric catalysis. Nat. Chem. 4, 603-614 (2012).

21. Lacour, J., Monchaud, D. \& Marsol, C. Effect of the medium on the oxaziridinium-catalyzed enantioselective epoxidation. Tetrahedron Lett. 43, 8257-8260 (2002).

22. Čorić, I. \& List, B. Asymmetric spiroacetalization catalysed by confined Brønsted acids. Nature 483, 315-319 (2012).

23. LaLonde, R., Wang, Z., Mba, M., Lackner, A. \& Toste, F. D. Gold (I)-catalyzed enantioselective synthesis of pyrazolidines, isoxazolidines, and tetrahydrooxazines. Angew. Chem. Int. Ed. 49, 598-601 (2010).

24. Mukherjee, S. \& List, B. Chiral counteranions in asymmetric transition-metal catalysis: highly enantioselective Pd/Brønsted acid-catalyzed direct a-allylation of aldehydes. J. Am. Chem. Soc. 129, 11336-11337 (2007).

25. Li, C., Wang, C., Villa-Marcos, B. \& Xiao, J. Chiral counteranion-aided asymmetric hydrogenation of acyclic imines. J. Am. Chem. Soc. 130, 14450-14451 (2008).

26. Hennecke, U., Müller, C. H. \& Fröhlich, R. Enantioselective haloetherification by asymmetric opening of meso-halonium ions. Org. Lett. 13, 860-863 (2011).

27. Liao, S. \& List, B. Asymmetric counteranion-directed transition-metal catalysis: enantioselective epoxidation of alkenes with manganese (III) salen phosphate complexes. Angew. Chem. Int. Ed. 49, 628631 (2010).

28. Merten, C., Pollok, C. H., Liao, S. \& List, B. Stereochemical communication within a chiral ion pair catalyst. Angew. Chem. Int. Ed. 54, 8841-8845 (2015).

29. Rutten, M. G. T. A., Vaandrager, F. W., Elemans, J. A. A. W. \& Nolte, R. J. M. Encoding information into polymers. Nat. Rev. Chem. 2,365-381 (2018).

30. Pizzolato, S. F., Štacko, P., Kistemaker, J. C., van Leeuwen, T. \& Feringa, B. L. Phosphoramiditebased photoresponsive ligands displaying multifold transfer of chirality in dynamic enantioselective metal catalysis. Nat. Catal. 3, 488-496 (2020). 
31. Feringa, B. L. The art of building small: from molecular switches to motors (Nobel lecture). Angew. Chem. Int. Ed. 56, 11060-11078 (2017).

32. Kistemaker, J. C., Pizzolato, S. F., van Leeuwen, T., Pijper, T. C. \& Feringa, B. L. Spectroscopic and theoretical identification of two thermal isomerization pathways for bistable chiral overcrowded alkenes. Chem. Eur. J. 22, 13478-13487 (2016).

33. Zhang, W., Loebach, J. L., Wilson, S. R. \& Jacobsen, E. N. Enantioselective epoxidation of unfunctionalized olefins catalyzed by salen manganese complexes. J. Am. Chem. Soc. 112, 2801-2803 (1990).

34. Irie, R., Noda, K., Ito, Y., Matsumoto, N. \& Katsuki, T. Catalytic asymmetric epoxidation of unfunctionalized olefins. Tetrahedron Lett. 31, 7345-7348 (1990).

35. Liao, S. \& List, B. Asymmetric counteranion-directed iron catalysis: a highly enantioselective sulfoxidation. Adv. Synth. Catal. 354, 2363-2367 (2012).

36. McGarrigle, E. M. \& Gilheany, D. G. Chromium- and manganese-salen promoted epoxidation of alkenes. Chem. Rev. 105, 1563-1602 (2005).

37. Vicario, J., Walko, M., Meetsma, A. \& Feringa, B. L. Fine tuning of the rotary motion by structural modification in light-driven unidirectional molecular motors. J. Am. Chem. Soc. 128, 5127-5135 (2006).

38. Štacko, P. et al. Locked synchronous rotor motion in a molecular motor. Science 356, 964-968 (2017).

39. Skarżewski, J., Gupta, A. \& Vogt, A. Influence of additional ligands on the two-phase epoxidation with sodium hypochlorite catalysed by (salen)manganese(III) complexes. J. Mol. Catal. Chem. 103, L63L68 (1995).

40. Jacobsen, E. N., Zhang, W., Muci, A. R., Ecker, J. R., Deng, L. Highly enantioselective epoxidation catalysts derived from 1,2-diaminocyclohexane. J. Am. Chem. Soc. 113, 7063-7064 (1991).

\section{Table 1}

Table 1 is available in the Supplementary Files section.

\section{Figures}


a<smiles>CC1CCc2ccc(O)c3c2c1c1c2ccccc2c2ccc(cc23)c2c(O)cccc21</smiles>

$\left(S, M, R_{\mathrm{a}}\right)$ b

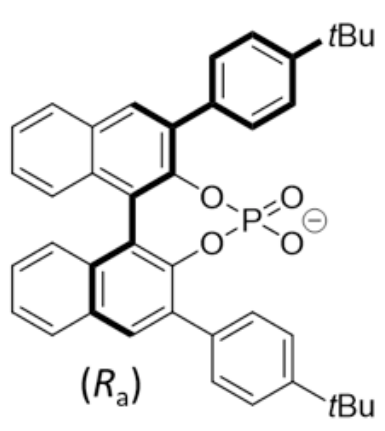

c

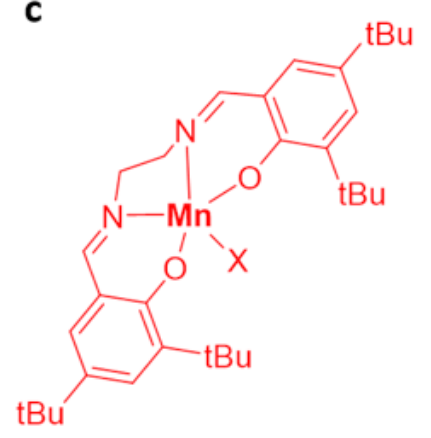

d

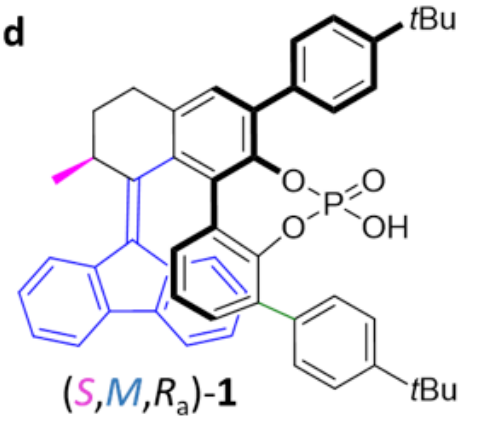

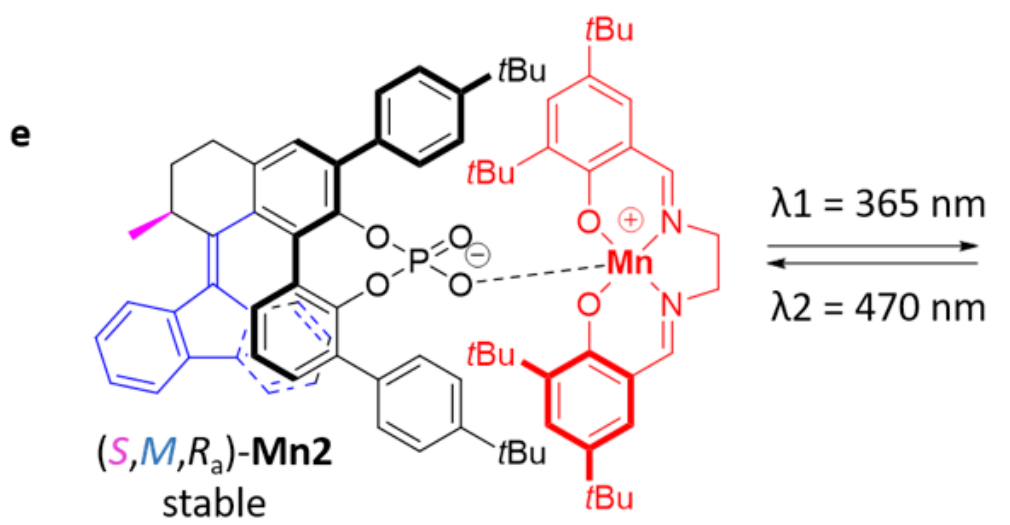

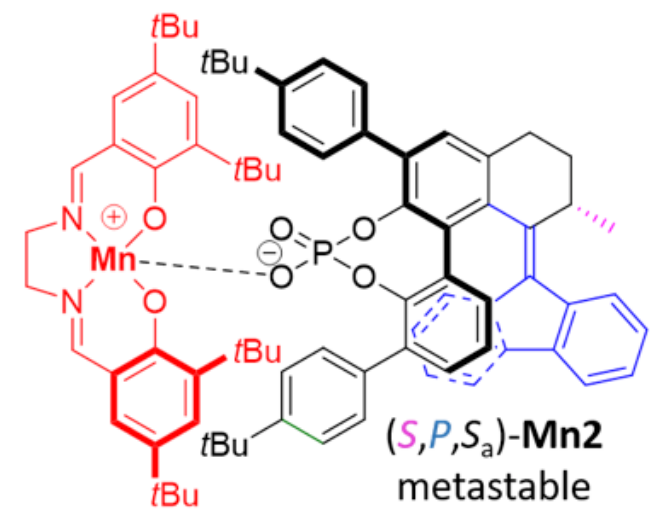

Figure 1

Structures of compounds. a Structure of a light-responsive 2,2'-biphenol-derived ligand based on a second-generation molecular motor. ${ }^{10} \mathbf{b}$ Structure of a bulky BINOL-derived phosphate anion. $\mathbf{c}$ Structure of a manganese(III) salen complex. $\mathbf{d}$ Structure of the molecular motor-based phosphoric acid. e Lightinduced switching between the pseudo-enantiomers of the ion-paired complexes $\left(S, M, R_{\mathrm{a}}\right)-\mathrm{Mn} 2$ and $\left(S, P, S_{\mathrm{a}}\right)-\mathrm{Mn} 2$. 

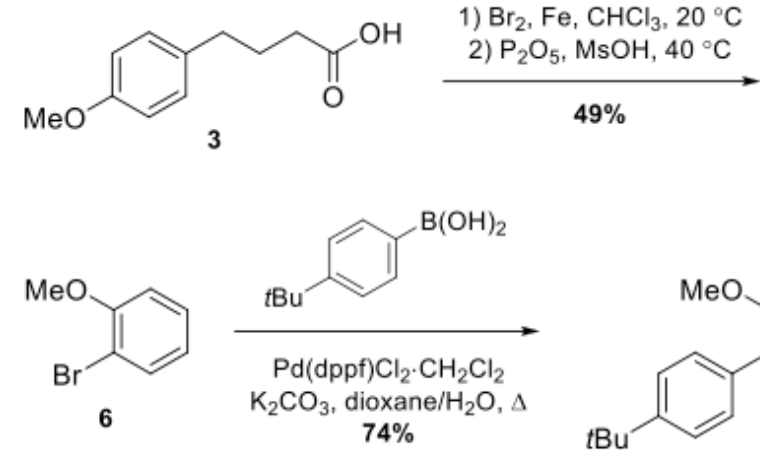

$74 \%$

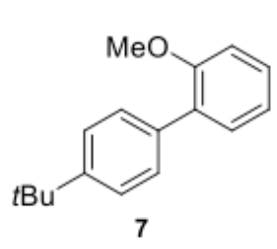

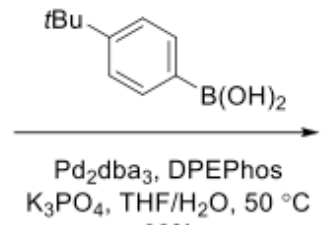

$68 \%$<smiles>COc1c(-c2ccc(C(C)(C)C)cc2)cc2c(c1Br)C(=O)CCC2</smiles>

5 4

$68 \%$

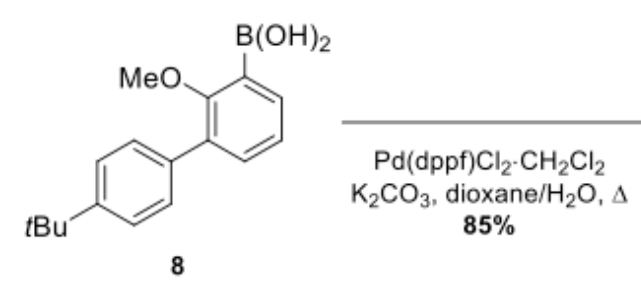<smiles>COc1c(-c2ccc(C(C)(C)C)cc2)cccc1-c1c(OC)c(-c2ccc(C(C)(C)C)cc2)cc2c1/C(=N/N)C(C)CC2</smiles><smiles>COc1c(-c2c(OC)c(-c3ccc(C(C)(C)C)cc3)cc3c2C(=O)C(C)CC3)cccc1C(C)(C)C</smiles>

1) LDA, THF, $-78^{\circ} \mathrm{C}$ 2) Mel, $-78 \rightarrow 20^{\circ} \mathrm{C}$

tBu

$95 \%$<smiles>COc1c(-c2ccc(C(C)(C)C)cc2)cccc1-c1c(-c2ccc(C(C)(C)C)cc2)cc2c(c1OC)C(=O)CCC2</smiles>

1) PIFA, DMF, $-50^{\circ} \mathrm{C}$

2)

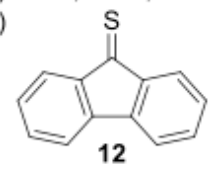

$-50 \rightarrow 20^{\circ} \mathrm{C}$

3) HMPT, PhMe, $\Delta$ $71 \%$<smiles>COc1ccccc1-c1c(-c2ccc(C(C)(C)C)cc2)cc2c3c1C(=C1c4ccccc4-c4ccccc41)C(C)CC3C2c1ccc(C(C)(C)C)cc1</smiles>

13<smiles></smiles>

14

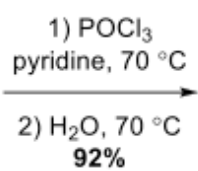

tBu<smiles></smiles>

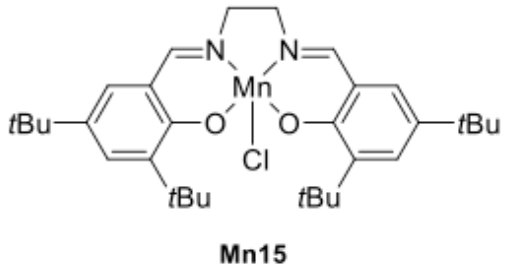

$\left(S, M, R_{\mathrm{a}}\right)-\mathbf{1}+\quad+\quad\left(R, P, S_{\mathrm{a}}\right)-\mathbf{1}$

first eluted second eluted

$\mathrm{Mn} 15, \mathrm{NaOH}$

acetone $20^{\circ} \mathrm{C}$

$\left(\mathrm{S}, \mathrm{M}, R_{\mathrm{a}}\right)-\mathrm{Mn} 2$

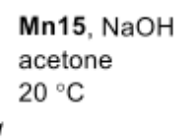

$\left(R, P, S_{\mathrm{a}}\right)-\mathrm{Mn} 2$
$\mathrm{Mn} 15, \mathrm{NaOH}$ acetone $20^{\circ} \mathrm{C}$

\section{Figure 2}

Synthesis and resolution of ion-paired switch-manganese(III) salen catalyst Mn2. (For clarity, only single enantiomers of 13, 14 and 1 are shown.) 
b
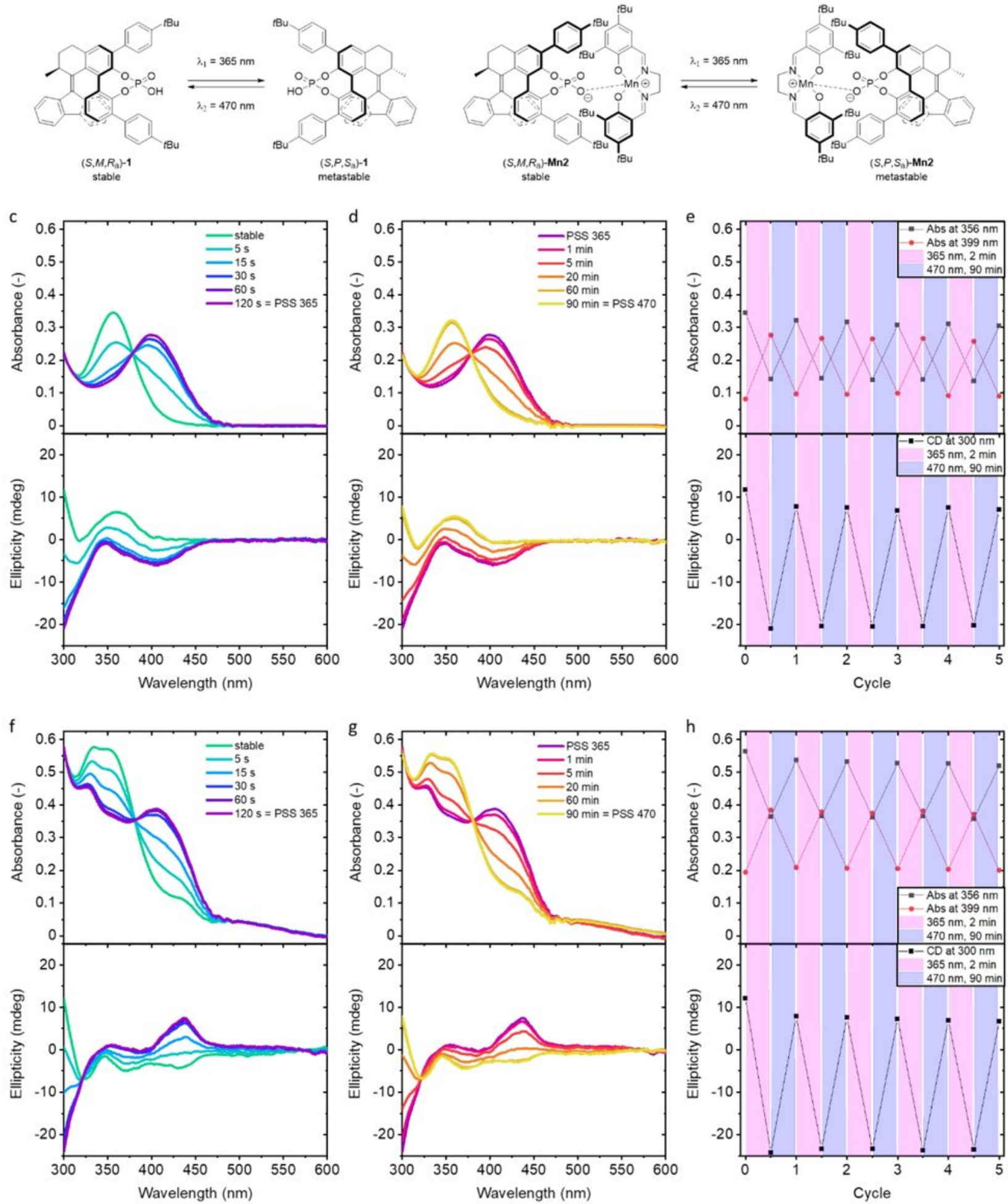

Figure 3

UV-vis and CD spectroscopic studies of the photochemical switching of $\left(S, M, R_{\mathrm{a}}\right)-1$ and $\left(S, M, R_{\mathrm{a}}\right)-\mathrm{Mn} 2$. a Scheme showing the reversible switching of 1. b Scheme showing the reversible switching of Mn2. c UVvis (top) and CD (bottom) spectra of $\left(S, M, R_{\mathrm{a}}\right)-1$ during irradiation with UV light $(\lambda=365 \mathrm{~nm})$. d UV-vis (top) and $\mathrm{CD}$ (bottom) spectra of $\left(S, M, R_{\mathrm{a}}\right)-1$ during switching from the metastable to the stable state 
upon irradiation with visible light $(\lambda=470 \mathrm{~nm})$. e Irradiation cycles of $\left(S, M, R_{\mathrm{a}}\right)-\mathbf{1}$ monitored with UV-vis (top) and CD spectroscopy (bottom). f UV-vis (top) and CD (bottom) spectra of $\left(S, M, R_{\mathrm{a}}\right)$-Mn2 during switching from the stable to the metastable state upon irradiation with UV light $(\lambda=365 \mathrm{~nm}) ; \mathbf{g} U V$-vis (top) and CD (bottom) spectra of $\left(S, M, R_{\mathrm{a}}\right)-\mathrm{Mn} 2$ during switching from the metastable to the stable state upon irradiation with visible light $(\lambda=470 \mathrm{~nm})$. $\mathbf{h}$ Irradiation cycles of $\left(S, M, R_{\mathrm{a}}\right)-\mathrm{Mn} 2$ monitored with UV-vis (top) and CD spectroscopy (bottom). For all experiments $\mathrm{c}=10^{-4} \mathrm{M}$ in benzene, $\mathrm{I}=2 \mathrm{~mm}$.

a
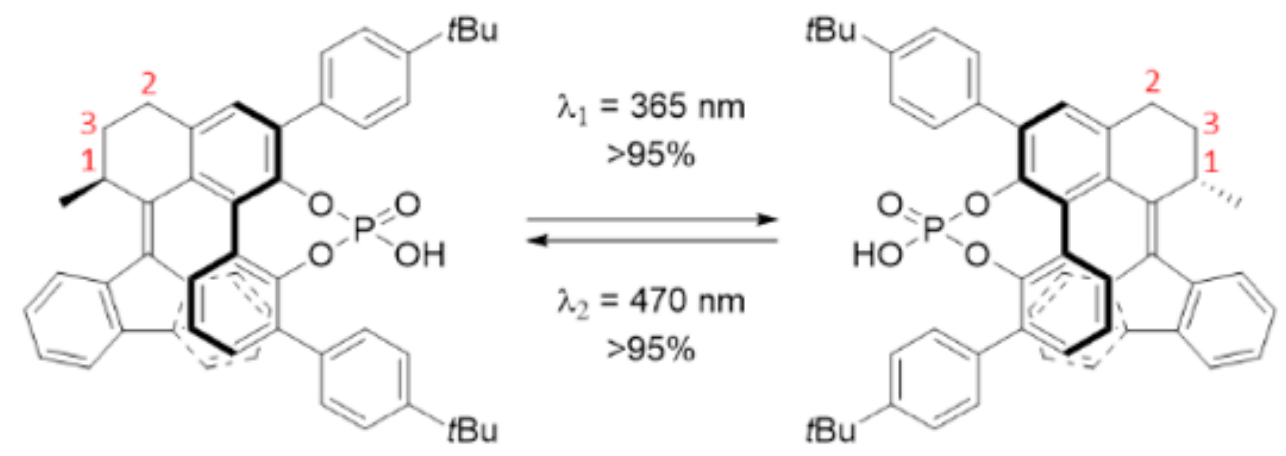

$\left(S, M, R_{\mathrm{a}}\right)-\mathbf{1}$

$\left(S, P, S_{\mathrm{a}}\right)-1$

stable

metastable

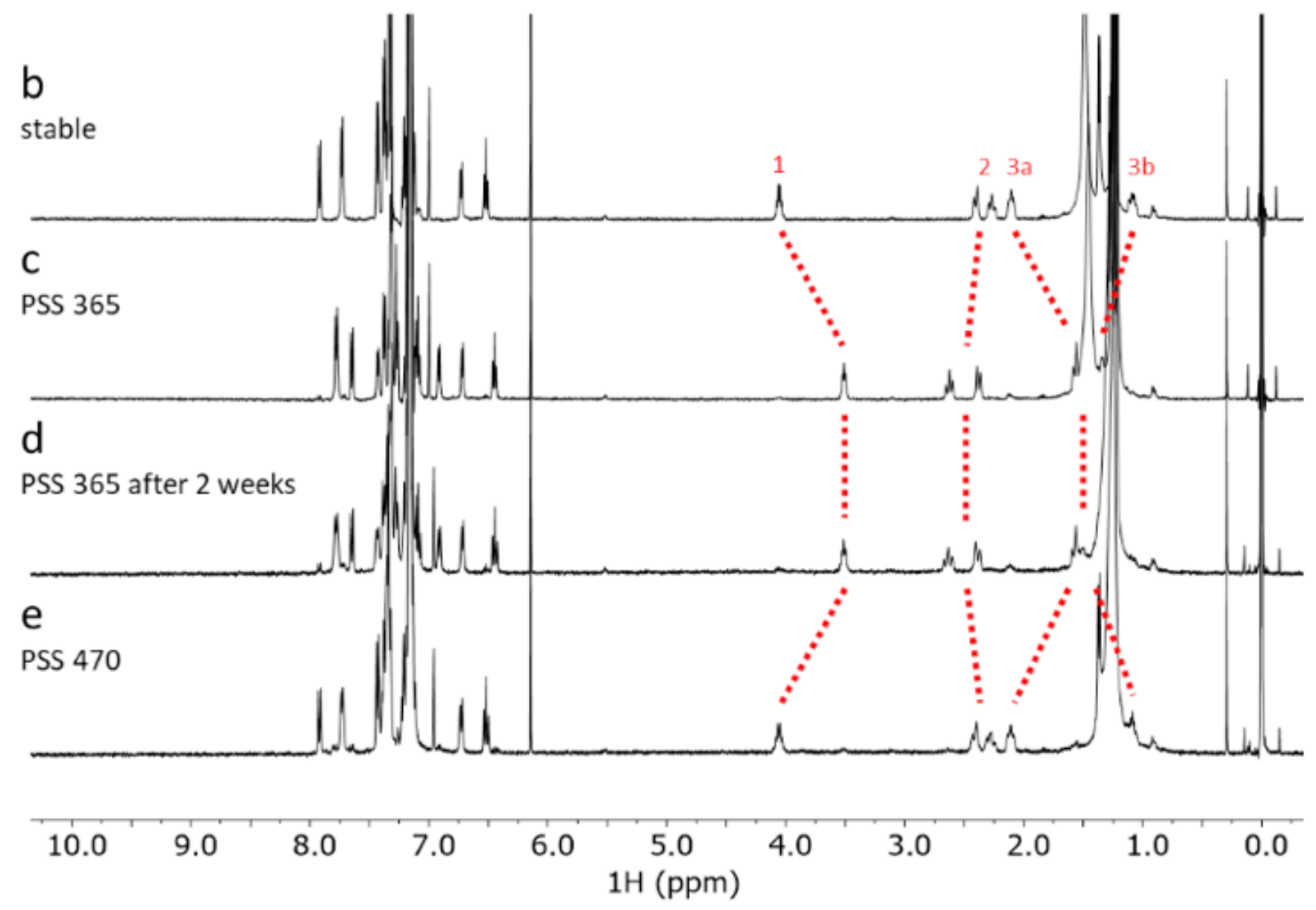


Figure 4

NMR study of the photochemical switching of rac-1. a Schematic representation of the reversible switching of 1 ; for clarity, only one of the enantiomers $\left(S, M, R_{\mathrm{a}}\right)$ is shown. b-e ${ }^{1} \mathrm{H}$ NMR spectra of rac-1 $\left(\mathrm{C}_{6} \mathrm{D}_{6}, 298 \mathrm{~K}, \mathrm{c}=2 \times 10^{-3} \mathrm{M}\right)$ : $\boldsymbol{b}$ in its stable state before irradiation; $\mathbf{c}$ after irradiation to the metastable state with UV light at $\lambda=365 \mathrm{~nm}$ for $45 \mathrm{~min}$; $\mathbf{d}$ after leaving the $\mathrm{PSS}_{365}$ mixture standing for 2 weeks; $\mathbf{e}$ after irradiation of the $\mathrm{PSS}_{365}$-mixture with visible light at $\lambda=470 \mathrm{~nm}$ for $18 \mathrm{~h}$, resulting in almost complete isomerization of rac-1 back to its stable state.

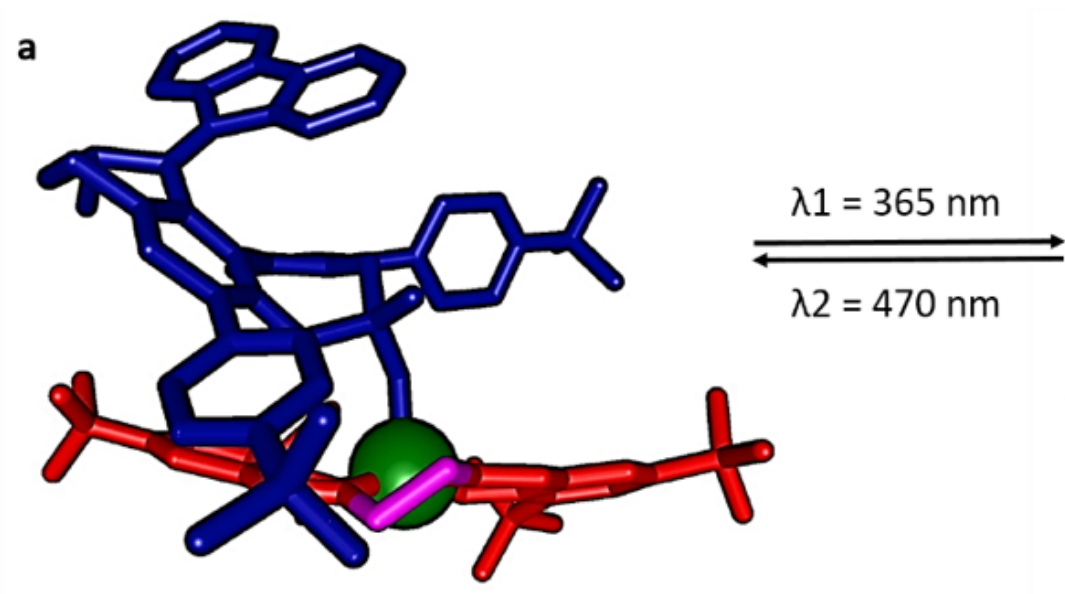

b

a

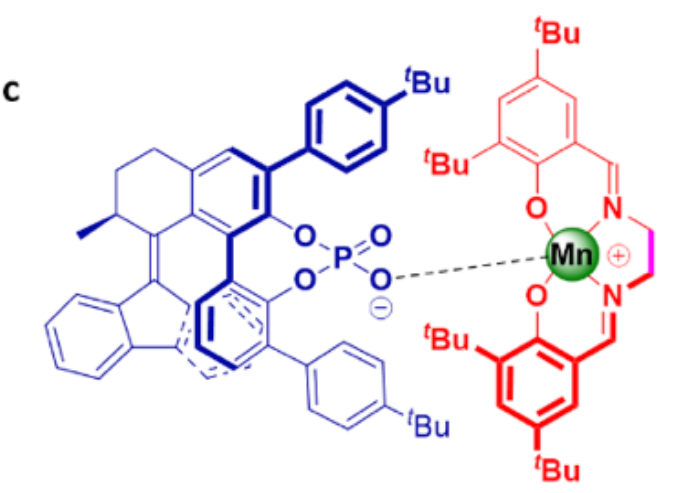
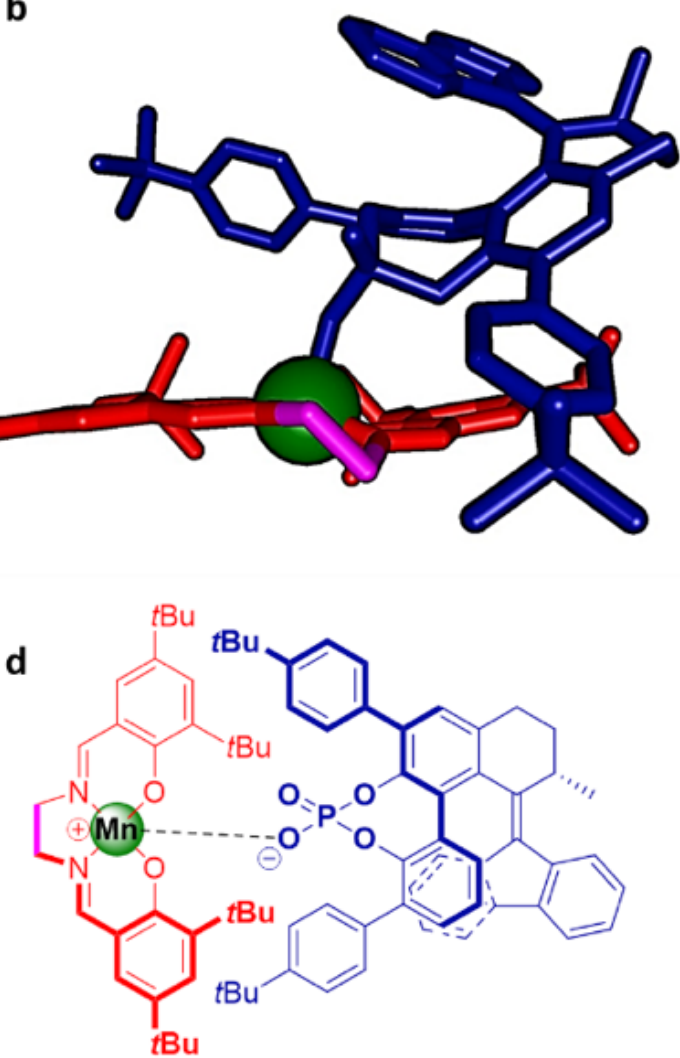

Figure 5

X-ray crystal structures of the stable and metastable forms of Mn2. a Front view of the X-ray structure of the stable $\left(S, M, R_{\mathrm{a}}\right)-\mathrm{Mn} 2$ isomer. $\mathbf{b}$ Front view of the X-ray structure of the metastable $\left(S, P, S_{\mathrm{a}}\right)-\mathrm{Mn} 2$ isomer. For ease of comparison in the same color coding: $\mathbf{c}$ the schematic structure of $\left(S, M, R_{\mathrm{a}}\right)-\mathrm{Mn} 2$ and $\mathbf{d}$ the schematic structure of $\left(S, P, S_{\mathrm{a}}\right)-\mathrm{Mn} 2$. Color coding: blue is phosphate ligand 1; red is salen ligand; magenta is ethylene bridge of salen ligand; green is manganese center; hydrogen atoms have been omitted for clarity. The single crystal structures shown in $\mathbf{a}$ and $\mathbf{b}$ were selected from the crystal structures of rac-Mn2 and irradiated rac-Mn2, respectively (for more detailed crystal data see Supplementary Information). 

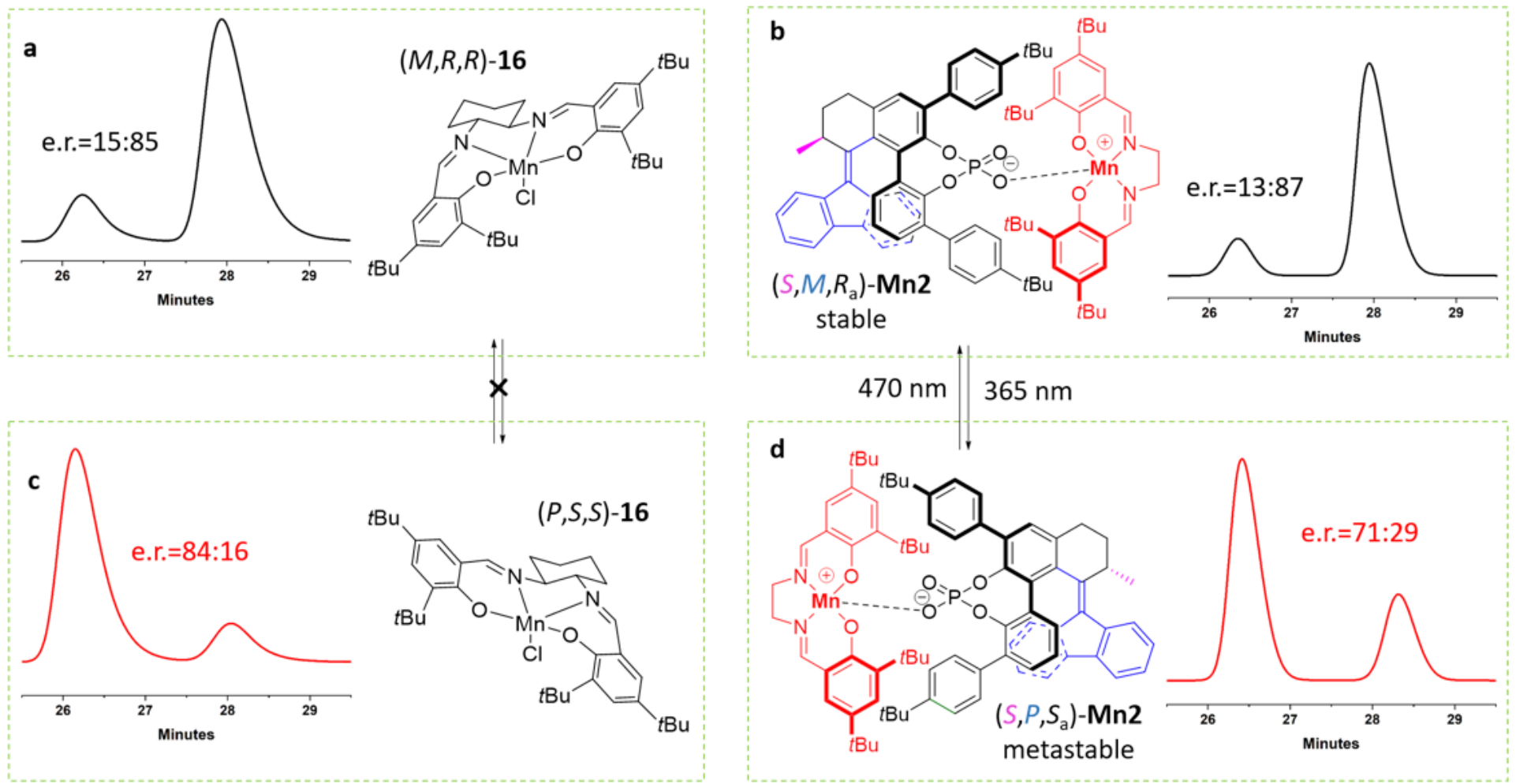

Figure 6

Enantioselective epoxidations. Enantioselective epoxidation of $\mathbf{1 7}$ and determination of the absolute configuration of the catalysts, based on the chiral HPLC traces of the product mixture of the epoxidation catalyzed by a $(M, R, R)-16, \mathbf{b}\left(S, M, R_{\mathrm{a}}\right)-\mathbf{M n} 2, \mathbf{c}(P, S, S)-16$ and $\mathbf{d}\left(S, P, S_{\mathrm{a}}\right)-\mathbf{M n} \mathbf{2}$.

\section{Supplementary Files}

This is a list of supplementary files associated with this preprint. Click to download.

- Table1.docx

- EnantiodivergentepoxidationSI.docx

- p2150anotforsubmission.cif

- p2125b.cif 